\title{
Remaining useful life prognostics for the electro-hydraulic actuator using relevance vector machine and optimized on-line incremental learning
}

\author{
Jiankang Dong ${ }^{1}$, Jiaqi Wang $^{2}$, and Runxia Guo ${ }^{1, *}$ \\ ${ }^{1}$ School of Electronic Information and Automation, Civil Aviation University of China, No. 2898 Jin \\ North Road, Tianjin, 300300, China \\ ${ }^{2}$ School of Computer science and technology,Civil Aviation University of China, No. 2898 Jin North \\ Road, Tianjin, 300300, China
}

\begin{abstract}
The electro-hydraulic actuator plays a significant role in the automatic flight control system, so it is vital to predict the remaining useful life (RUL) for the electro-hydraulic actuators. Relevance vector machine (RVM) is flourishing in the field of RUL prognostics and gradually applied to the prediction of complex systems or components, but the general RVM cannot achieve on-line prediction efficiently due to its high computational complexity, besides, the sparse RVM model which is only based on historical data set could cause a large prediction error in the long term. To deal with these plights, an optimized incremental learning algorithm based on RVM is presented taking full advantage of the on-line updating samples to improve the precision of prognostics.
\end{abstract}

\section{Introduction}

Electro-hydraulic actuator is a core component in the Automatic Flight Control System (AFCS) of civil aircraft. Failures in electro-hydraulic actuator could lead to loss of operation, functional degradation, machine halt and even catastrophic failure [1]. Therefore, the reliability of electro-hydraulic actuator becomes a subject of great interest to the civil aircraft avionics system and predict the remaining useful life (RUL) in an effective way are imperative [2]. Figure 1 shows a typical simplified diagram of an electro-hydraulic actuator.

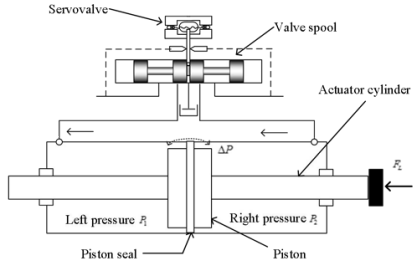

Fig. 1. Schematic of the electro-hydraulic actuator.

\footnotetext{
* Corresponding author: rxguoblp@163.com
} 
In the existing literature, the prognostics approaches for RUL can be broadly divided into model-based approaches and data-driven approaches. Model-based approaches like Kalman filtering (KF) and particle filtering (PF) typically involve the establishment of a physical model to describe the mechanism of the system degradation and failure evolvement. Therefore, they may not be suitable for many practical applications [3]. Contrarily, data-driven approaches which are used to capture the inherent relationships could avoid the dependence on the system's analytical model, thus they can extract essential information from historical and monitoring data without modelling. Therefore, data-driven approaches have gradually became the primary methodology in the field of prognostics due to the easy-to-use and flexible modelling characteristics [4].

Thus far, the prognostics based on RVM algorithm is generally off-line which does not possess dynamic updating capability of the model and has low prediction precision in longterm [5]. Particularly, the RVM algorithm equipped with the characteristic of small sample size still has no efficacious on-line training technology that can be actualized to process online modelling and prognostics [6]. To overcome these defects, this paper presents an optimized on-line incremental learning algorithm based on RVM to realize electrohydraulic actuator's RUL prognostics. In the proposed method, the RVM algorithm is herein applied to predict the RUL of electro-hydraulic actuator by capturing the correspondence between the hydraulic difference and sample entropy, in addition, the uncertainty of the predictive results is also characterized.

\section{Reviews of related theories}

\subsection{Relevance vector machine}

The RVM introduces a prior to the weight of the model and the most probable value is iteratively estimated from the data set [7]. Given a set of examples of input vectors $\left\{x_{i}\right\}_{i=1}^{N}$ and their corresponding target vectors $\left\{t_{i}\right\}_{i=1}^{N}$. The purpose is to learn a dependency model between the targets and inputs vectors for the sake of making precise prognostics value of $t_{i}$ for unseen value of $x_{i}$ [8]. Generally, the predictions are based on a function $y(x)$ defined over the input space and the function $y(x)$ can be defined as below:

$$
y\left(x_{i} ; \omega\right)=\sum_{i=0}^{N} \omega_{i} K\left(x, x_{i}\right)
$$

where $\omega=\left[\omega_{0}, \omega_{1}, \cdots, \omega_{N}\right]^{T}$ denotes the weight vector, $K\left(x, x_{i}\right)$ is a kernel function.

RVM seems to predict target $t_{i}$ according to

$$
t_{i}=y\left(x_{i} ; \omega\right)+\varepsilon_{i}
$$

where $\varepsilon_{i}$ is an independent sample from noise process with mean 0 and variance $\sigma^{2}$.

The likelihood estimation of all the sampled data sets can be written as (3)

$$
P\left(t \mid \omega, \sigma^{2}\right)=\left(2 \pi \sigma^{2}\right)^{-N / 2} \exp \left\{-\left(2 \sigma^{2}\right)^{-1}\|-\Phi \omega\|^{2}\right\}
$$

$$
\begin{aligned}
& \text { where } \quad \Phi=\left[\varphi\left(x_{1}\right), \varphi\left(x_{2}\right), \ldots, \varphi\left(x_{N}\right)\right]^{T} \quad \text { is } \quad \text { an } \quad N \times(N+1) \quad \text { design } \quad \text { matrix, } \\
& \varphi\left(x_{i}\right)=\left[1, K\left(x_{i}, x_{1}\right), K\left(x_{i}, x_{2}\right), \ldots, K\left(x_{i}, x_{N}\right)\right]^{T} .
\end{aligned}
$$

Given a new input $x^{*}$, its corresponding output probability distribution is

$$
P\left(t^{*} \mid t, \alpha_{M P}, \sigma_{M P}^{2}\right)=N\left(t^{*} \mid y^{*}, \sigma_{*}^{2}\right)
$$


where the mean and the variance are respectively $y^{*}=\mu^{T} \phi\left(x^{*}\right), \sigma_{*}^{2}=\sigma_{M P}^{2}+\phi\left(x^{*}\right)^{T} \Sigma \phi\left(x^{*}\right)$.

\subsection{Sample entropy}

In this paper, the sample entropy is represented by a symbol $E_{\text {samp }}$. Here, using $E_{\text {samp }}$ to process the original voltage sequence can greatly reduce the training samples, which provide potential for high computational efficiency of on-line prediction.

For a given $N_{c}$ total number of data points, we form the $N_{c}-m+1$ vectors $x_{m}(p)$ as

$$
x_{m}(p)=[x(p), x(p+1), \ldots, x(p+m-1)], 1 \leq p \leq N_{c}-m+1
$$

where $m$ is the length of a subseries of the dataset $N_{c}$.

The distance between two such vectors and is defined as follow

$$
d_{m}\left[x_{m}(p), x_{m}(q)\right]=\max \left[\left\|x_{m}(p+k)-x_{m}(q+k)\right\|\right], 0 \leq k \leq m-1
$$

The $E_{\text {samp }}$ is ultimately estimated by

$$
E_{\text {samp }}=-\ln \left[\frac{A^{m}(r)}{B^{m}(r)}\right]
$$

where $B^{m}(r)$ and $A^{m}(r)$ represent the probabilities that two sequences match for $m$ and $m+1$ points, respectively.

\section{Method for the RUL prognostics of electro-hydraulic actuator}

\subsection{Data preprocessing}

Sampled $N$ total number cycles of voltage sequence $\left\{x_{m}(p)\right\}_{p=1}^{N_{c}}$ from an electro-hydraulic actuator and each cycle contains $N_{c}$ data points, then compute the $E_{\text {samp }}$ of voltage sequence for each cycle. Then the new data set $\left\{E_{\text {samp }}(i)\right\}_{i=1}^{N}$ representing a sample entropy vector is employed as data input and the corresponding hydraulic difference vector $\{\Delta P(i)\}_{i=1}^{N}$ as target vector of RVM, respectively.

$$
\Delta P(i)=\left\|P_{1}(i)-P_{2}(i)\right\|
$$

where $P_{1}(i)$ represents the pressure on the left of the cylinder, $P_{2}(i)$ stands for the pressure on the right of the cylinder in Figure 1.

\subsection{Training algorithm for RVM}

In this section, an RVM prognostics model is built by training the newly obtained sample entropy data set. The steps for training RVM model are as follows:

Step 1. Initialization: initialize the weight $\omega$ and variance $\sigma^{2}$.

Step 2. Expectation step: utilize $\omega^{(\kappa)}$ and $\left(\sigma^{2}\right)^{\kappa}$ to estimate the $(\kappa+1)$ th iteration and

$$
\begin{gathered}
E\left(\omega \omega^{T}\right) \cdot \omega^{(\kappa+1)}=\left(\sigma^{-2}\right)^{(\kappa)}\left(\Psi^{(\kappa)}-\Psi^{(\kappa)} \Phi^{T} \Lambda^{-1} \Phi \Psi^{(\kappa)}\right) \Phi^{T} t \\
E\left(\omega \omega^{T}\right)=\left(\Psi^{(\kappa)}-\Psi^{(\kappa)} \Phi^{T} \Lambda^{-1} \Phi \Psi^{(\kappa)}\right)+\omega \omega^{T}
\end{gathered}
$$


where $\Lambda=\Phi \Psi^{(\kappa)} \Phi^{T}+\left(\sigma^{2}\right) I, \quad \Psi^{(\kappa)}=\operatorname{diag}\left[\left|\omega_{0}^{(\kappa)}\right|^{2},\left|\omega_{1}^{(\kappa)}\right|^{2}, \ldots,\left|\omega_{N}^{(\kappa)}\right|^{2}\right] \quad$ and $\quad \omega^{(\kappa)} \quad, \quad \omega^{(\kappa+1)}$ represent the weights at iterations $\kappa$ and $\kappa+1$.

Step 3. Maximization step: calculate the new variance according to $\omega^{(\kappa+1)}$ obtained in Step 2.

Step 4. Convergence judgment: stop the iteration if $\left\|\omega^{(\kappa+1)}-\omega^{(\kappa)}\right\| /\left\|\omega^{(\kappa)}\right\|<\delta$ or the algorithm reaches the maximum iteration number, or else go to Step 2 to start a new iteration.

\subsection{Updating on-line model with optimized incremental learning algorithm}

The incremental learning algorithm is adopted in this paper with the merit of learning new knowledge without forgetting previously learned. However, with the on-line new data updating, the size of on-line training samples will increase gradually, which could lead to low computational efficiency. Therefore, a new optimized algorithm is proposed in this paper to improve the calculation efficiency of on-line prognostics. Detailed steps are shown in Figure 2.

Take $\tau_{0}$ cycle as the start point of the prognostics, predict the hydraulic difference $\Delta P(i)$ using the initial model until the predicted value reaches the failure threshold $U$, then the real hydraulic difference values will obtain one by one during this process. After a $l$-step prediction stage, the prognostics precision should be checked. Adopt the real hydraulic difference values of the $l$-step (from cycle $\tau_{0}+1$ to $\tau_{0}+l$ ) to update the corresponding predictive values and add them to the previous training samples. Set an updating threshold $T$ to determine whether to update the current model or not. Compute the correlation coefficient $(C)$ of the two time series before and after the updating, if the result outweighs the updating threshold, it indicates that the newly added samples have little influence on the current model and there is a good accordance between the predictive and actual values, so the model does not need to be updated. Continue predicting and adding the next $l$-step's real hydraulic difference values, compute the correlation coefficient until the calculation result is less than the updating threshold, then update the current model using the latest training samples and continue to predict with the new model at cycle $\tau_{1}$. Repeat the above steps.

The correlation coefficient $(C)$ is defined as follow:

$$
C=\frac{\sum_{i=1}^{l}\left(y_{i}-\bar{y}\right)\left(\hat{y}_{i}-\overline{\hat{y}}\right)}{\sqrt{\sum_{i=1}^{l}\left(y_{i}-\bar{y}\right)^{2}} \sqrt{\sum_{i=1}^{l}\left(\hat{y}_{i}-\overline{\hat{y}}\right)^{2}}}
$$

where $y_{i}$ is the actual value, $\hat{y}_{i}$ is the predictive value, $\bar{y}$ is the mean of actual values, and $\overline{\hat{y}}$ is the mean of predictive values, $l$ is the length of a prediction stage.

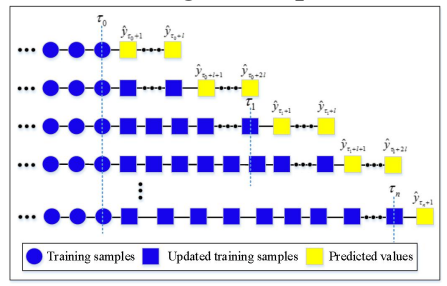

Fig. 2. Basic schematic of online model updating. 


\subsection{RUL}

The prognostics stops and the remaining useful life (RUL) is calculated at cycle $\tau_{\text {end }}$ when the failure threshold of the electro-hydraulic actuator met. The failure threshold can be considered to occur when the predictive hydraulic difference degradation value drops to $70 \%$ of its initial value in actual application, and the RUL at the current cycle can be obtained by measuring the distance between the cycle $\tau_{\text {end }}$ and the cycle $\tau_{0}$ at which the prediction starts. The predicted RUL is given as:

$$
R U L=\tau_{\text {end }}-\tau_{0}
$$

where $\tau_{\text {end }}$ is the intersection of the prognostics curve and the failure threshold line.

\section{Experiment and analysis}

In order to further verify the performance of the proposed on-line RUL prognostics algorithm, we conduct an experiment against the internal leakage fault of an electrohydraulic actuator. The voltage data set is selected from an electro-hydraulic actuator, which has nominal voltage of $115 \mathrm{~V}$ with $400 \mathrm{HZ}$ and the maximum hydraulic difference of 2200psi. The actual life of the electro-hydraulic actuator is about one million cycles. In order to accelerate the results of the experiment, the electro-hydraulic actuator experiment is conducted with the 720 times' acceleration to simulate run-to-failure test. The experiment performed on the data set at three different prognostics start cycles, which are $30 \%$ end of life (EOL), 45\% EOL and 60\% EOL, respectively. EOL represents the failure cycle where the hydraulic difference reaches the failure threshold defined as $800 \mathrm{psi}$ in this study. Under the 720 times simulation accelerated run-to-failure test, the actual cycle life of the electrohydraulic actuator is 1400 cycles, and a cycle refers to the time length of an actuator cylinder swinging back and forth.

\subsection{Case study of the proposed RUL prognostics method}

In this study, the start points of the data set at 30\% EOL, $45 \%$ EOL and $60 \%$ EOL are 420 cycles, 630 cycles and 840 cycles, respectively. The failure threshold takes at 1400 cycles and the time interval $\Delta T$ of a cycle is set $2.89 \mathrm{~s}$. $l$ represented the length of a prediction stage is set through the experience and the suitable one namely 30 is chosen to conduct this experiment.

In order to fully validate the superiority of the proposed optimized incremental RVM (optimized I-RVM) prognostics algorithm, Figure 3 shows the process of updating the prognostics model constantly and the 13 different RUL results predicted by different updated models indicate that the more times the model is updated, the higher the prognostics accuracy is.

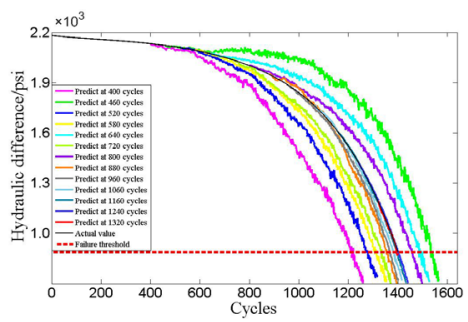

Fig. 3. The process of updating the prognostics model. 
The tracks of the RUL predicted by original RVM, retraining RVM (R-RVM), incremental RVM (I-RVM) and optimized incremental RVM (optimized I-RVM) at 30\% EOL, 45\% EOL and 60\% EOL are shown in Figure 4, respectively.

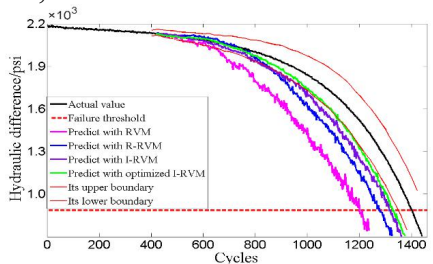

(a)

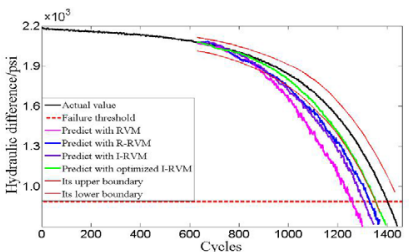

(b)

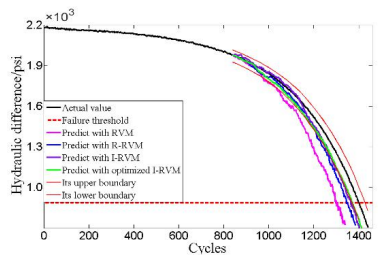

(c)

Fig. 4. The comparison results of the proposed different methods at different start cycles (a): at $30 \%$ EOL (b): at $45 \%$ EOL (c): at $60 \%$ EOL.

Table 1 shows the predict performance comparison of the three methods.

Table 1. Predict performance comparison of the three methods.

\begin{tabular}{|c|c|c|c|}
\hline Algorithm & Start points & RUL error (cycle) & Time consumption (s) \\
\hline RVM & $30 \%$ EOL & 189 & $1.465 \pm 0.043$ \\
\hline R-RVM & $30 \%$ EOL & 161 & $7.812 \pm 0.121$ \\
\hline I-RVM & $30 \%$ EOL & 140 & $5.657 \pm 0.052$ \\
\hline optimized I-RVM & $30 \%$ EOL & 132 & $4.579 \pm 0.040$ \\
\hline RVM & $45 \%$ EOL & 165 & $1.960 \pm 0.037$ \\
\hline R-RVM & $45 \%$ EOL & 149 & $7.281 \pm 0.156$ \\
\hline I-RVM & $45 \%$ EOL & 118 & $5.268 \pm 0.049$ \\
\hline optimized I-RVM & $45 \%$ EOL & 76 & $4.023 \pm 0.038$ \\
\hline RVM & $60 \%$ EOL & 101 & $2.595 \pm 0.045$ \\
\hline R-RVM & $60 \%$ EOL & 63 & $6.915 \pm 0.146$ \\
\hline I-RVM & $60 \%$ EOL & 45 & $5.024 \pm 0.036$ \\
\hline optimized I-RVM & $60 \%$ EOL & 36 & $3.652 \pm 0.035$ \\
\hline
\end{tabular}

It can be seen from Figure 4 and Table 1 that the predicted RUL results with the proposed optimized I-RVM prognostics mothed are almost close to the actual values and have the minimum error value all the time.

\section{Conclusion}

In this paper, a novel on-line RUL prognostics algorithm for electro-hydraulic actuator has been proposed in view of the problem that RVM can not realize on-line prediction. The incremental learning algorithm realizes the on-line prognostics and improves the performance of RVM long-time prediction. The optimized I-RVM method has greatly reduced the training samples and improved the computational efficiency by using sample entropy to process the original voltage data set. The experimental comparison results show a satisfactory performance of the optimized I-RVM on-line prognostics method in terms of prediction efficiency accuracy and computational efficiency, which may act as a further reference for other similar domains related to remaining useful life prognostics and real time condition monitoring.

\section{Acknowledgements}

This work was jointly supported by the National Natural Science Foundation of China (Grant 61603395); the National Natural Science Foundation of China and the Civil 
Aviation Administration of China (Grant U1433103); the National Key R \& D Project of China (Grant 2016YFB0502405); the Special Program of Talents Development for Excellent Youth Scholars in Tianjin.

\section{References}

1. Li H, Pan D and Chen C L P 2014 Intelligent Prognostics for Battery Health Monitoring Using the Mean Entropy and Relevance Vector Machine IEEE Trans. Systems Man \& Cybernetics Systems. 44 851-862

2. Hu X, Jiang J, Cao D and Bo E 2016 Battery Health Prognosis for Electric Vehicles Using Sample Entropy and Sparse Bayesian Predictive Modeling IRE Trans. Ind. Electron. 63 2645-56.

3. Guo R, Wang J, Zhang N and Dong J 2018 State prediction for the actuators of civil aircraft based on a fusion framework of relevance vector machine and autoregressive integrated moving average J. Syst. Control Eng. 1-13

4. Liu D, Wang H, Peng Y, Xie W and Liao H 2013 Satellite Lithium-Ion Battery Remaining Cycle Life Prediction with Novel Indirect Health Indicator Extraction Energies. 6 3654-68

5. Liu D, Peng Y, Li J and Peng X 2013 Multiple optimized online support vector regression for adaptive time series prediction Measurement. 46 2391-404

6. Chen C, Kunche S, and Pecht M 2013 Incremental learning approach for improved prediction IEEE Int. Conf. Prognostics and Health Management. pp 1-7

7. Lou J, Jiang Y, Shen Q, Shen Z and Wang Z 2015 Software reliability prediction via relevance vector regression Neurocomputing. 66-73

8. Widodo A, Shim M C, Caesarendra W and Yang B S 2011 Intelligent prognostics for battery health monitoring based on sample entropy Expert Systems with Applications 38 11763-69 